\title{
Wave interactions in a shallow-water model
}

\author{
K. Wiklund \\ Department of Plasma Physics. Umeå University, S-90187 Umeå. Sweden
}

Received: 10 September 1998 - Accepted: 2 March 1999

\begin{abstract}
By using a Hamiltonian method, non-lincar three-wave interaction in a class of systems related to the shallow water model is considered and a general coupling coefficient is presented. In the special casc where two inertial waves and one Rossby wave interact resonantly, it is found that even a very small shear of the background velocity can be important in the interaction process. The stability of the system is considered by using a pseudoenergy method. Some implications for the dynamics of atmospheric flows are pointed out.
\end{abstract}

\section{Introduction}

Observations of large scale atmospheric and ocean motions have shown that the energy spectra have a peak close to the Coriolis frequency $\mathrm{f}=2 \Omega \sin \theta$ where $\Omega$ is the rotation frequency of the planet and $\theta$ is the latitude (see e.g. the review by Van Delden 1992). Since the frequencies of weakly nonlinear inertial waves in a shallow water system, which is a model often used in geophysics, are close to the frequency f (e.g. Petviashvili and Pokhotelov 1992) it would be of interest to know how these inertial waves couple with themselves and with other waves. This coupling could for example be a mechanism of energy transfer between low and high-frequency waves. The most simple nonlinear interaction occurs when three waves interact resonantly, i.e. when the frequencies of the waves satisfy the frequency matching condition $\omega_{1}+\omega_{2}+\omega_{3}=0$ (e.g. Weiland and Wilhelmsson 1977 , Craik 1985). Self-interaction of three inertial waves is then out of question since the resonance condition above cannot be satisfied. Thus we have to focus attention on the coupling between a combination of inertial waves and some other types of waves. One example of such a combination, which we will consider in this paper, is the

Correspondence to : $\mathrm{K}$. Wiklund resonant interaction between two inertial waves and one Rossby wave (which has a frequency much less than f). We consequently deduce the equations governing the evolution of these three interacting waves. The mathematical properties of such equations have been studied extensively (e.g. Weiland and Wilhelmsson 1977, Craik 1985) and arc thus wcll known.

The equations of shallow water have a deep analogy with those of drift vortices in magnetised plasmas (c.g. Nezlin and Chernikov 1995; Horton and Ichikawa 1996). This implies, for example, that one may use devices devoted to hydrodynamic experiments in order to get some understanding of the equivalent phenomena in plasma physics, and vice versa. The common way to analyse the shallow-water equations is to reduce them, by the quasigeostrophic approximation, to the Charney equation or to the quasi-geostrophic potential vorticity equation (Horton and Ichikawa 1996). However, the importance of going beyond this approximation to include further aspects of the full nonlinear shallow-water equations has been pointed out by Nezlin and Chernikov (1995). The latter authors have shown that some of the interesting dynamics, e.g. the existence of monopolar solutions, disappears in the standard approximation schemes and that it thus is necessary to study a generalised Charney equation. Since, in this paper, we are concerned with the interaction properties of inertial waves we cannot adopt the quasiqeostrophic approximation because this approximation filters out the inertial waves (e.g. Salmon 1988, Petviashvili and Pokhotelov 1992), and thus we will not use the Charney equation. It should be noticed, however, that other kinds of three-wave interactions can be described by the Charney equations.

The canonical Hamiltonian method (Zakharov 1971, 1984) has been a very fruitful way to analyse the lhuid dynamics. Using that method it can, however, be difficult to find the canonical variables and to interpret these variables physically. If, for example, we want to use the non-canonical variables velocity, density and entropy to describe an ordinary fluid, we cannot apply the canonical 
method (Morrison 1998). In order to use the physical variables of a system we thus need to consider a noncanonical approach. In this paper we shall analyse the full shallow-water equations by such a non-canonical Hamiltonian perturbation method. This has previously been done for both plasma and lluid systems (Axelsson 1998; Larsson 1996,1998a,b). Thus the method yields the governing equations of a non-linear resonant three-wave interaction process wherc the coupling coefficients between the waves are expressed explicitly. The method also provides us with conserved quantities that can be used in a stability-analysis corresponding to the Lyapunov stability theory, i.c. we can obtain conditions on an unperturbed state such that any perturbation remains in the neighbourhood of the unperturbed state. The unperturbed state considered in this paper is a zonal flow (the background velocity is parallel to the equator and depends only on the latitude). Such flows have for example been observed by Voyager in Jupiters atmosphere and in the monsoons of the Earth's atmosphere (Petviashvili and Pokhotelov 1992, Dowling 1995). In a zonal flow, or shear flow, we have the possibility of negative energy modes which can affect the stability of the system (KelvinHelmholtz instability). The stability and flow properties in case of a shear flow have previously been discussed for many different systems (e.g. Craik 1985, Fabrikant and Stepanyants 1998). In this paper we want to show how a sheared background flow affects the non-linear resonant three-wave interaction process and how one should choose the background flow in order to avoid explosive instabilities.

In section 2 the model equations are described. In section 3 we present them in a noncanonical Hamiltonian form and give a definition of what we mean with a Hamiltonian system. Section 4 is concerned with the nonlinear threewave interaction, and we deduce the explicit coupling coefficients. In section 5 we present a stability criterion obtained by the Hamiltonian method used in this paper.

\section{The model equations}

We will use a mathematical model which has the same structure as that of a shallow water system. However, our model contains a class of systems and the interpretation of the variables depends on the choice of system. In the examples below we have presented two different ways to interpret the model equations. By using our approach we will be able to obtain results for a class of systems simultaneously, and it may also help us to see similarities between apparently different systems. The model equations are

$\partial_{i} \mathbf{v}=-\mathbf{v} \cdot \nabla \mathbf{v}-\nabla P+f \mathbf{v} \times \hat{\mathbf{z}}$

and

$\partial_{r} H=-\nabla \cdot[H \mathbf{v}]$ where the variable $\mathbf{v}$ typically represents a fluid velocity, and $\mathbf{H}$ could be viewed as a density-like variable, or the height of the fluid. A specific model is obtained from (1) and (2) by a choice of internal energy $U=U(H, x, y)$ and a Coriolis-like function $\mathrm{f}=\mathrm{f}(\mathrm{x}, \mathrm{y})$. The generalised pressure $P=P(H, x, y)$, appearing in (1), is then defined as $P \equiv \partial_{I I} U$. Two examples below will show a specific interpretation of (1) and (2):

\section{Example I}

By choosing $U=1 / 2 g H^{2}$ and $H=H_{0}(x)+h(x, y, t)$, where $g$ is a constant, we obtain the ordinary shallow water system where $\mathrm{P}=\mathrm{gH}$ is the pressure and $\mathrm{f}=\mathrm{f}(\mathrm{x})$ is the Coriolis parameter. Those equations describe a lluid with an undisturbed layer with depth $H_{0}(x)$ and a perturbed layer with depth $h(x, y, t)$ where $H_{0} \gg h$. The fluid velocity $\mathbf{v}=\mathbf{v}(x, y)$ is in the $\mathrm{x}-\mathrm{y}$ plane and the system is thus quasi-two-dimensional.

\section{Example 2}

As another example we choose

$$
U=\frac{T}{M}\left[\ln \left(\frac{H}{H_{0}}\right)-1\right] H \text { and get } P=\frac{T}{M} \ln \left(\frac{H}{H_{0}}\right) .
$$

Here $\mathrm{T} / \mathrm{M}$ is the temperature to mass ratio. By letling $\mathbf{v} \rightarrow \mathbf{v}_{i}, f \rightarrow \omega_{c i}, H \rightarrow n$ and $H_{0} \rightarrow n_{0}$ the system now describes a nonuniform collisionless electron-ion plasma where $\mathbf{v}_{i}$ is the ion fluid velocity, $\omega_{c i}$ is the ion gyrofrequency and the ion density $\mathrm{n}$ is described by the Boltzman distribution. We assume quasi-neutrality here.

In the rest of the paper a general pressure $P$ is assumed if not stated otherwise.

\section{The Hamiltonian method}

Many non-dissipative systems, e.g. ideal fluids, MHDfluids and Vlasov systems in plasmas, have been shown to admit a Hamiltonian formulation. Thus we will, in this paper, use a Hamiltonian approach.

The system (1) and (2) can be shown to be Hamiltonian in the sense that the equations of motion can be written as

$\partial_{t} u=X(u)=J_{u} \frac{\delta H}{\delta u}$

where $\boldsymbol{H}$ is the Hamiltonian function and $\boldsymbol{J}$ is any operator that satisfies

a) the anti-symmetry condition

$\left\langle a, J_{u} b\right\rangle=-\left\langle J_{u} a, b\right\rangle$

and 
b) the Jacobi-like identity

$$
\begin{array}{r}
\left\langle J_{u} a, \frac{\delta}{\delta u}\left\langle b, J_{u} c\right\rangle\right\rangle+\left\langle J_{u} b, \frac{\delta}{\delta u}\left\langle c, J_{u} a\right\rangle\right\rangle \\
+\left\langle J_{u} c, \frac{\delta}{\delta u}\left\langle a, J_{u} b\right\rangle\right\rangle=0
\end{array}
$$

where $\langle$,$\rangle stands for the inner product and \mathrm{a}, \mathrm{b}$ and $\mathrm{c}$ are arbitrary operator arguments.

When we put (1) and (2) in the form (3) we obtain the following expressions

$u=\left(\begin{array}{c}\mathbf{v} \\ H\end{array}\right), \quad J_{u}=\left(\begin{array}{cc}-H^{-1}(f \hat{\mathbf{z}}+\nabla \times \mathbf{v}) \times & -\nabla \\ -\nabla . & 0\end{array}\right)$,

$H=\int\left(\frac{1}{2} H v^{2}+U\right) d x d y$

and $\frac{\delta \boldsymbol{H}}{\delta u}=\left(\begin{array}{c}H \mathbf{v} \\ \frac{1}{2} \mathrm{v}^{2}+P\end{array}\right)$

where we have chosen the variables $\mathbf{v}$ and $\mathrm{H}$ to be our state variables and the operator $J_{u}$ to satisfy the conditions above. It should here be stressed that the operator $J_{u}$ does not involve the pressure $P$. Since the whole Hamiltonian formulation is based on the structure of $J_{u}$ we thus can bc sure that changing the pressure model will not affect the Hamiltonian structure of the system. We have also delined the inner product as

$\langle a, b\rangle \equiv \int\left(\mathbf{a} \cdot \mathbf{b}+a_{4} b_{4}\right) d x d y$ where

$a=\left(\begin{array}{l}\mathbf{a} \\ a_{4}\end{array}\right)$ and $b=\left(\begin{array}{l}\mathbf{b} \\ b_{4}\end{array}\right)$.

Since our approach in this paper is to consider a Hamiltonian perturbation method we need to introduce the definitions of the perturbed objects. Let us thus introduce the following Taylor expansions of $\mathrm{X}, \mathrm{J}$ and $\mathrm{P}$

$X\left(u_{0}+\delta u\right)=X\left(u_{0}\right)+X_{u_{0}}^{(1)} \delta u+\frac{1}{2 !} X_{u_{0}}^{(2)}(\delta u, \delta u)+\ldots$

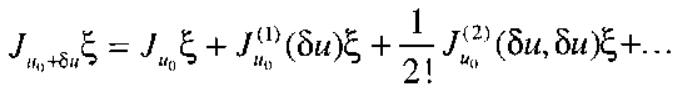

$P\left(H_{0}+h\right)=P\left(H_{0}\right)+P^{(1)}(h)+\frac{1}{2 !} P^{(2)}(h, h)+\ldots$

where $u_{0}$, representing the unperturbed statc, is a solution of the equations of motion, i.e. $\partial_{t} u_{0}=X\left(u_{0}\right)$. One explicit solution is $u_{0}=\left(\mathbf{v}_{0}, H_{0}\right)$ where $H_{0}(x)$ is an arbitrary function and $\mathbf{v}_{0}$ is the stationary sheared background velocity $\mathbf{v}_{0}=\mathrm{v}_{0}(x) \hat{\mathbf{y}}$ determined from the unperturbed equation $\nabla P\left(H_{0}\right)=f(x) \mathbf{v}_{0}(x) \times \hat{\mathbf{z}}$. Thus by specifying $P=P\left(H_{0}\right), H_{0}(x)$ and $\mathrm{f}(\mathrm{x})$ we determine the background flow $\mathbf{v}_{0}=\mathrm{v}_{0}(x) \hat{\mathbf{y}}$.

The equation of motion for a perturbation obtained from (1), (2) and (4) is

$\partial_{t} \delta u=X_{u_{0}}^{(1)} \delta u+\frac{1}{2 !} X_{u_{u}}^{(2)}(\delta u, \delta u)+\frac{1}{3 !} X_{u_{u_{0}}}^{(3)}(\delta u, \delta u, \delta u)+\ldots$

where only the first two terms are needed in this paper

$X_{u_{0}}^{(1)} a=\left(\begin{array}{c}-\mathbf{v}_{0} \cdot \nabla \mathbf{a}-\mathbf{a} \cdot \nabla \mathbf{v}_{0}-\nabla P^{(1)}\left(a_{4}\right)+f \mathbf{a} \times \hat{\mathbf{z}} \\ -\nabla \cdot\left(H_{0} \mathbf{a}\right)-\mathbf{v}_{0} \cdot \nabla a_{4}\end{array}\right)$

and

$X_{u_{0}}^{(2)}(a, a)=2\left(\begin{array}{c}-\mathbf{a} \cdot \nabla \mathbf{a}-\nabla P^{(2)}\left(a_{4}, a_{4}\right) \\ -\nabla \cdot\left(a_{4} \mathbf{a}\right)\end{array}\right)$.

From (4) we also obtain the operator

$J_{u_{w},}=\left(\begin{array}{cc}-\frac{R}{H_{0}} \hat{\mathbf{z}} \times & -\nabla \\ -\nabla & 0\end{array}\right)$ where $R \equiv f+\mathrm{v}_{0}^{\prime}$.

The operator $J_{u_{0}}$ is the Hamiltonian structure for the linearisation of (3).

We consider dynamical accessible (or Hamiltonian) perturbations from the background state, i.e. perturbations that in principle may be obtained by a change in the Hamiltonian (e.g. Morrison 1998, Larsson 1998b). The Hamiltonian perturbations should to first order satisfy (Larsson 1998b)

$$
\begin{aligned}
& \delta u=J_{u_{0}} \xi \\
& \partial_{t} \xi=-X_{u_{0}}^{(1)^{*} \xi} \\
& \partial_{t} \delta u=X_{u_{11}}^{(1)}(\delta u)
\end{aligned}
$$

where $X_{u_{0}}^{(1)^{*}}$ is defined from $\left\langle a, X_{u_{b}}^{(1)} b\right\rangle=\left\langle X_{u_{k j}}^{(1)^{*}} a, b\right\rangle$ and the generating function $\xi$ is the conjugate-variable. By operating with $J_{u_{0}}$ on (6) and using (5) we note that solutions of (6) give us solutions of (7) if (5) is satisfied.

\section{Wave coupling}

The non-linear coupling between the waves can be an efficient mechanism of transfering energy between different parts of the energy spectrum. The procedure to derive the coupling equations bctween three resonant modes is rather standard (e.g Craik 1985). Let us first briefly recapitulate it in order to introduce the reader to the notations. 
Let us consider an interaction, with a stationary background, between three linearly independent resonant normal modes $\xi_{a}, \xi_{b}$ and $\xi_{c}$ satisfying

$\partial_{t} u_{0}=0, X\left(u_{0}\right)=0$,

$X_{u_{0}}^{(1)^{*}} \xi_{j}=i \omega_{j} \xi_{j}$

and the resonance condition

$\omega_{a}+\omega_{b}+\omega_{c}=0$

where $j=a, b, c$

The three waves are considered as Hamiltonian perturbations of the background state, i.e.

$u_{j}=J_{0} \xi_{j}$

which implies that $X_{u_{0}}^{(1)} u_{j}=-i \omega_{j} u_{j}$.

By using the Ansatz

$u=u_{0}+\sum_{j=a}^{c}\left[C_{j}(t) \tilde{u}_{j} e^{-i \omega_{j} t}+c . c.\right]$

where $u_{j}=\tilde{u}_{j}(x) \exp \left(-i \omega_{j} t\right)$ and writing

$\partial_{t} \delta u=X_{u_{0}}^{(1)} \delta u+\frac{1}{2 !} X_{u_{0}}^{(2)}(\delta u, \delta u)$

we obtain the equations describing the coupling between the slowly varying amplitudes C (Larsson 1996)

$$
\begin{aligned}
& \frac{d}{d t} \bar{C}_{a}=-i \omega_{a} \frac{V}{W_{a}} C_{b} C_{c} \\
& \frac{d}{d t} \bar{C}_{b}=-i \omega_{b} \frac{V}{W_{b}} C_{c} C_{a} \\
& \frac{d}{d t} \bar{C}_{c}=-i \omega_{c} \frac{V}{W_{c}} C_{a} C_{b}
\end{aligned}
$$

where $W_{j} \equiv-\left\langle\bar{\xi}_{j}, X_{u_{0}}^{(1)} u_{j}\right\rangle$

$V \equiv-\left\langle\xi_{a}, X_{u_{0}}^{(2)}\left(u_{b}, u_{c}\right)\right\rangle$

and the overbar denotes the complex conjugate.

The coupling coefficient $\mathrm{V}$ is, from general theory (Larsson 1998a,b), symmetric in the indices a, b and $c$. The expression $W_{j}\left|C_{j}\right|^{2}$ is what most authors denote by pseudo-energy or quasi-energy. We shall investigate it more closely in section 5 where also the stability is discussed.

\subsection{General coupling coefficient}

Since the coupling coefficients describes how strongly the waves are coupled to each other, it is important to derive an explicit expression where the effect of for example a sheared background flow could be seen. Thus, let us first cxamine the coupling coefficient (16) and then at the end of this section derive a dispersion relation.

Writing the perturbations as $\mathbf{v}=\mathbf{w}(x) \exp (i \theta)$ and $h=s(x) \exp (i \theta)$ with $\theta=k_{y} y-\omega t$, we thus introduce for each variable the real quantities amplitude and phase. The interaction is most efficient when the resonance condition

$k_{y u}+k_{y h}+k_{y c}=0$

is satisfied, and we assume that this is the case here (Weiland and Wilhelmsson 1977). By substituting the explicit cxpressions from section 3 into (16), using the linear relations (5)-(7) and assuming that all lields approach zero at infinity, we obtain, after some lengthy but straightlorward algebra, the following general expression for the coupling coefficient

$$
\begin{aligned}
& V=\int\left[h_{a} \mathbf{v}_{b} \cdot \mathbf{v}_{c}+h_{b} \mathbf{v}_{c} \cdot \mathbf{v}_{a}+h_{c} \mathbf{v}_{a} \cdot \mathbf{v}_{b}\right] d x d y \\
& +\int R \hat{\mathbf{z}} \cdot\left\{\frac{\mathbf{G}^{\text {hut }}}{\hat{\omega}_{a} \hat{\omega}_{h}}-\frac{\mathbf{G}^{\mathrm{cah}}}{\hat{\omega}_{n} \hat{\omega}_{c}}\right\} d x d y+ \\
& +\int \frac{H_{0} \mathrm{v}_{0}^{\prime} R}{\hat{\omega}_{a}}\left\{-\frac{v_{x b} k_{y a}}{\hat{\omega}_{a} \hat{\omega}_{b}} f_{a t c}-\frac{v_{x c} k_{y i}}{\hat{\omega}_{a t} \hat{\omega}_{c}} f_{a b}\right. \\
& \left.-\frac{v_{x b} k_{y b}}{\hat{\omega}_{b}, \hat{\omega}_{b}} f_{u i c}-\frac{v_{x c} k_{y c}}{\hat{\omega}_{c} \hat{\omega}_{c}} f_{c t b}\right\} d x d y \\
& +2 \int i H_{0}^{2} \mathrm{v}_{0}^{\prime} v_{x i t} v_{x i}, v_{x c} \partial_{x}\left(\frac{R}{H_{0} \hat{\omega}_{a i} \hat{\omega}_{b} \hat{\omega}_{c}}\right) d x d y \\
& +\int h_{t}\left\{P^{(2)}\left(h_{b}+h_{c}, h_{b}+h_{c}\right)\right. \\
& \left.-P^{(2)}\left(h_{b}, h_{b}\right)-P^{(2)}\left(h_{c}, h_{c}\right)\right\} d x d y
\end{aligned}
$$

where we have used the compact notation

$$
\begin{aligned}
& \mathbf{G}^{b k a} \equiv \mathbf{g}_{b} \times \nabla \times\left(H_{0} \mathbf{g}_{c} \times \mathbf{g}_{a}\right), \\
&-\mathbf{g}_{c} \times \mathbf{g}_{a} \nabla \cdot\left(H_{0} \mathbf{g}_{b}\right) \\
& \mathbf{g}_{j} \equiv \mathbf{v}_{j}-\frac{i \mathbf{v}_{0}^{\prime} v_{x j}}{\hat{\omega}_{j}} \hat{\mathbf{y}}, \\
& \mathrm{v}_{0}^{\prime} \equiv \partial_{x} \mathbf{v}_{0}, f_{a b} \equiv \hat{\mathbf{z}} \cdot \mathbf{v}_{a} \times \mathbf{v}_{b} \text { and } \\
& \hat{\omega}_{j} \equiv k_{y j} \mathbf{v}_{0}-\omega_{j} .
\end{aligned}
$$

It follows from (19) that $\mathbf{G}^{a b c}+\mathbf{G}^{b c a}+\mathbf{G}^{c a b}=0$.

The first and fourth term in (18) is obviously symmetric. After some algebra it can be shown that also the second and third terms arc symmetric. Note that we have not yet 
specified which model we use, i.e. $P^{(2)}$ is not defined. Thus next step is therefore to specify our model. However, it is instructive to first examine what types of waves we have in our general model.

The coupling coefficient (18) can obviously be expressed only in $h(x, y)$ by using the relations

$$
\begin{aligned}
\mathbf{v}=\frac{1}{\hat{\omega}^{2}-f R}[ & i \hat{\omega} \nabla P^{(1)}(h) \\
& \left.+f \nabla P^{(1)}(h) \times \hat{\mathbf{z}}-\mathrm{v}_{\hat{\partial}}^{\prime} \partial_{x} P^{(1)}(h) \hat{\mathbf{y}}\right]
\end{aligned}
$$

and

$h=\frac{i \nabla \cdot\left(H_{0} \mathbf{v}\right)}{\hat{\omega}}$

which are obtained from (5),(7) and (17).

If we substitute (22) into (23) and usc (17) we will end up with a differential equation in $s(x)$ which, if solved, gives us the dispersion relation

$s^{\prime \prime}+G_{1} s^{\prime}+G_{0} s=0$

where

$$
\begin{aligned}
G_{1} & \equiv \partial_{x} \ln \left(\frac{H_{0}}{\hat{\omega}^{2}-f R}\right)+\frac{\partial_{x} P_{0}^{\prime}}{P_{0}^{\prime}}+\frac{f}{i \hat{\omega}} \frac{\partial_{y} P_{0}^{\prime}}{P_{0}^{\prime}}, \\
G_{0} & \equiv\left(\frac{f k_{y}}{\hat{\omega}} \partial_{x} \ln \left(\frac{H_{0} f}{\hat{\omega}^{2}-f R}\right)-k_{y}^{2}+\frac{\hat{\omega}^{2}-f R}{H_{0} P_{0}^{\prime}}\right) \\
& +\frac{\partial_{x}^{2} P_{0}^{\prime}}{P_{0}^{\prime}}+\frac{\partial_{x}\left(f \partial_{y} P_{0}^{\prime}\right)}{i \hat{\omega} P_{0}^{\prime}}+i k_{y} \frac{\partial_{y} P_{0}^{\prime}}{P_{0}^{\prime}}
\end{aligned}
$$

and $P^{(1)}(h)=P_{0}^{\prime} h$.

Thus, if $s$ is constant, we note that $G_{0}=0$ which in case of shallow water $\left(P^{(1)}(h)=g h\right)$ implies that

$$
\hat{\omega}\left[1+r_{0}^{2} k_{y}^{2}-\frac{\hat{\omega}^{2}}{f^{2}}+\frac{\mathrm{v}_{0}^{\prime}}{f}\right]=k_{y} \frac{g H_{0}}{f} \partial_{x} \ln \left(\frac{H_{0} f}{\hat{\omega}^{2}-f R}\right)
$$

where $r_{0} \equiv\left(g H_{0}\right)^{1 / 2} / f$ is the Rossby radius.

The dispersion relation (25) contains three different types of waves

Rossby waves $(\omega \ll f)$ :

$$
\hat{\omega} \approx \frac{k_{y} \mathrm{v}_{R}}{1+r_{0}^{2} k_{y}^{2}+\frac{\mathrm{v}_{0}^{\prime}}{f}}
$$

where $\quad \mathrm{v}_{R} \equiv \frac{g H_{0}}{f} \partial_{x} \ln \left(\frac{H_{0}}{R}\right)$

Inertial waves $(\omega-f)$ :

$\hat{\omega}^{2} \approx f^{2}\left(1+r_{0}^{2} k_{y}^{2}\right)+f v_{0}^{\prime}$ and

Gravity waves $(\omega \gg f)$ :

$\hat{\omega}^{2} \approx f^{2} r_{0}^{2} k_{y}^{2}+f v_{0}^{\prime}$

Rossby waves, which are due to the inhomogenity of the Coriolis frequency $\mathrm{f}$, are analogous to drift waves in plasmas. At much higher frequencies we have the inertial waves which are analogous to cyclotron waves in plasmas. Finally we have the gravity waves, or the shallow water gravity waves, which sometimes behave like sound waves.

\subsection{Two inertial waves and one Rossby wave}

In this section we assume that $P=g H$ (shallow water) which implies that $P^{(2)}(h, h)=0$ and that the last term in (18) is zero.

Here we will take a look at the special case when one wave is a Rossby wave with frequency $\hat{\omega}_{a} \ll f$ and the other two are inertial waves with frequencies $\hat{\omega}_{b}-\hat{\omega}_{c}-f$ (Pokhotelov et al., 1995;1996).

Let us consider the case where $\mathrm{v}_{0}^{\prime} \leq f$ and where we also assume that $H_{0} k_{y a} \ll 1$ and $f \ll 1$. It is obvious from the dispersion relations (26) and (27), together with the resonance conditions $(10)$ and (17), that all $k_{y}$ must be of the same order of magnitude, i.e. $k_{y a}-k_{y b}-k_{y c}$.

A comparison of the four remaining terms in (18) shows that the sum of term two and term three dominates in both of the two approximation regimes $s_{j}^{\prime} \ll k_{y j} s_{j}$ and $s_{j}^{\prime}-k_{y j} s_{j}$ where $\mathrm{j}=\mathrm{a}, \mathrm{b}, \mathrm{c}$. The first (second) regime corresponds to the case when the inhomogenity-scale of $\mathrm{s}(\mathrm{x})$ is much larger than (of the same order as) the wavelength. Thus the general coupling coefficient (18) is approximated by

$$
\begin{aligned}
& V=\int R \hat{\mathbf{z}} \cdot\left\{\frac{\mathbf{G}^{b c a}}{\hat{\omega}_{a} \hat{\omega}_{b}}-\frac{\mathbf{G}^{c a t}}{\hat{\omega}_{a} \hat{\omega}_{c}}\right\} d x d y \\
& +\int \frac{H_{0} \mathrm{v}_{0}^{\prime} R}{\hat{\omega}_{a}}\left\{-\frac{v_{x}^{b} k_{y a}}{\hat{\omega}_{a} \hat{\omega}_{b}} f_{a c}-\frac{v_{x}^{c} k_{y a}}{\hat{\omega}_{a} \hat{\omega}_{c}} f_{u b}\right\} d x d y
\end{aligned}
$$

where we also have used $\hat{\omega}_{a} \ll \hat{\omega}_{b}-\hat{\omega}_{c}$ and $k_{y a} \sim k_{y b}-k_{y c}$ in the third term in (18).

In order to obtain a more explicit expression, without using the compact notation introduced carlier, we substitute (22) into (29). After some algebra, where we used (24) and the condition $\hat{\omega}_{a} \ll f-\hat{\omega}_{b} \sim \hat{\omega}_{c}$, we obtain 


$$
\begin{aligned}
& V=-\int i K^{u t k} \frac{R H_{0}}{\hat{\omega}_{a} \hat{\omega}_{b}}\left[\frac{H_{0}^{\prime}}{H_{0}}-\frac{R}{R}\right]\left(f s_{b}+\hat{\omega}_{h} \frac{s_{b}^{\prime}}{k_{y^{\prime}}}\right) . \\
& \left\{\hat{\omega}_{c}\left(R \frac{s_{a}^{\prime} s_{c}^{\prime}}{k_{y a} k_{y c}}-f s_{u t} s_{c}\right)+f R\left(\frac{s_{u}^{\prime}}{k_{y a}} s_{c}-s_{a} \frac{s_{c}^{\prime}}{k_{y c}}\right)\right\} d x \\
& -\int i K^{a b c} \frac{R H_{0}}{\hat{\omega}_{a} \hat{\omega}_{c}}\left[\frac{H_{0}^{\prime}}{H_{0}}-\frac{R^{\prime}}{R}\right]\left(f s_{c}+\hat{\omega}_{c} \frac{s_{c}^{\prime}}{k_{y c}}\right) \text {. } \\
& \left\{\hat{\omega}_{b}\left(R \frac{s_{a}^{\prime} s_{b}^{\prime}}{k_{y a} k_{y b}}-f_{s_{a}} s_{b}\right)+f R\left(\frac{s_{a}^{\prime}}{k_{y a}} s_{b}-s_{a} \frac{s_{b}^{\prime}}{k_{y b}}\right)\right\} d x \\
& +\int i K^{a b c} \frac{R H_{0}}{\hat{\omega}_{a} \hat{\omega}_{b}}\left[\frac{H_{0}^{\prime}}{H_{0}}-\frac{R^{\prime}}{R}+\frac{\hat{\omega}_{u t}^{\prime}}{\hat{\omega}_{a}}\right] \text {. } \\
& \left\{f s_{b}+\hat{\omega}_{b} \frac{s_{b}^{\prime}}{k_{y b}}\right) \frac{f v_{0}^{\prime}}{\hat{\omega}_{a}}\left\{f s_{a} s_{c}+\hat{\omega}_{c} s_{a} \frac{s_{c}^{\prime}}{k_{y c}}\right\} d x \\
& +\int i K^{a b c} \frac{R H_{0}}{\hat{\omega}_{a} \hat{\omega}_{c}}\left[\frac{H_{0}^{\prime}}{H_{0}}-\frac{R^{\prime}}{R}+\frac{\hat{\omega}_{a i}^{\prime}}{\hat{\omega}_{a}}\right] \text {. } \\
& \left(f s_{c}+\hat{\omega}_{c} \frac{s_{c}^{\prime}}{k_{y c}}\right) \frac{f v_{0}^{\prime}}{\hat{\omega}_{a}}\left\{f s_{a} s_{b}+\hat{\omega}_{b}, s_{a} \frac{s_{b}^{\prime}}{k_{y b}}\right\} d x
\end{aligned}
$$

where $K^{a b c} \equiv \frac{i g k_{y a}}{\hat{\omega}_{a}^{2}-f R} \frac{i g k_{y b}}{\hat{\omega}_{b}^{2}-f R} \frac{i g k_{y c}}{\hat{\omega}_{c}^{2}-f R}$.

Here it should be stressed that (30) is valid in both approximation regimes $s_{j}^{\prime} \ll k_{y j} s_{j}$ and $s_{j}^{\prime}-k_{y j} s_{j}$.

The expression (30) above seems to be just as complicated as (18) and one could wonder what we have gained. The point is however that in (18) we have used a compact notation which is not transparent to the reader, and difficult to evaluale for specified cases. The expression (30), although rather lenghty, does not contain any operators, however. Each term in (30) is of a very simple form and it is thus easy to compare the different terms with each other and to estimate numerical values for them. If we now further assume that $s_{j}^{\prime} \ll k_{y j} s_{j}$ the coupling coefficient will reduce to

$$
\begin{aligned}
V & =\int i K^{u d x} s_{u} s_{b} s_{c} \frac{f^{2} R H_{0}}{\hat{\omega}_{a}}\left[\frac{H_{0}^{\prime}}{H_{0}}-\frac{R^{\prime}}{R}\right]\left\{\frac{\hat{\omega}_{c}}{\hat{\omega}_{b}}+\frac{\hat{\omega}_{b}}{\hat{\omega}_{c}}\right\} d x \\
& -\int i K^{u t x} s_{a} s_{b} s_{c} \frac{f^{2} R H_{0}}{\hat{\omega}_{a}}\left[\frac{H_{0}^{\prime}}{H_{0}}-\frac{R^{\prime}}{R}+\frac{\hat{\omega}_{a}^{\prime}}{\hat{\omega}_{a}}\right] \frac{f \mathrm{v}_{0}^{\prime}}{\hat{\omega}_{b} \hat{\omega}_{c}} d x
\end{aligned}
$$

In (31) only the second term involves the shear of the background velocity $v_{0}^{\prime}$. This term will be of the same order as the first term if $\mathrm{v}_{0}^{\prime} \sim f$. By examining (30) instead of (31) we can obtain a similar result. Thus we can conclude that the shear of the background velocity is important within the regime of approximation considered in this section.

As an example we consider wave motion in the atmosphere of the Earth choosing the following typical values on the parameters: $\omega_{a}-10^{-6} s^{-1}, \omega_{b} \sim \omega_{c}-f-10^{-4} s^{-1}$,

$f^{\prime} \sim 10^{-11} m^{-1} s^{-1}, H_{0}-10^{3} m, g-10 m s^{-2}$

By using the unperturbed equation

$g H_{0}^{\prime}(x)=f(x) \mathrm{v}_{0}(x)$ we have $\mathrm{v}_{0}^{\prime} \sim\left(10^{9} H_{0}^{\prime \prime}-10^{-3} \mathrm{v}_{0}\right) f$ and thus the importance of shear (i.e. the condition $\mathrm{v}_{0}^{\prime} \sim f$ ) depends sensitively on the $\mathrm{x}$-dependence of $H_{0}(x)$.

Choosing the model $H_{0}=H_{00}+x^{2} / l$ and

$h_{j}(x, y)=s_{0 j} \exp \left(-x^{2} / L^{2}\right) \exp \left(i k_{y j} y-i \omega_{j} t\right)$

with

$H_{00}-10^{3} \mathrm{~m}, l-10^{9} \mathrm{~m}$ and $L \sim 10^{5} \mathrm{~m}$ we obtain from (31) and (32) $V \sim 10^{10} s_{0 a} s_{0 b} s_{0 c}$ and $W_{j}-10^{13} s_{0 j}^{2}$ where $\mathrm{j}=\mathrm{a}, \mathrm{b}, \mathrm{c}$. This means that $V / W_{j}-10^{-3} s_{0 i f} s_{0 b} s_{0 c} / s_{0 j}^{2}$, i.e. the growth rates in the coupling equations (14) may be of order $10^{-3}$ times the incrtial wave frequency.

\section{Stability}

Conserved quantities are uscful for considering stability by the Lyapunov method. The conservation laws obtained from the noncanonical Hamiltonian formulation (3) (Larsson 1998b) may then be used. One of these is the encrgy related quantity $\left\langle\xi, X_{u_{6}}^{(1)}(u)\right\rangle$ which is exactly conserved by (5)-(7). If this is a delinite (positively or negatively) quantity then at lcast linear stability is indicated.

By substituting the explicit expression of $X_{t t_{0}}^{(1)}$ from section 3 into (15), and by using the relations (5)-(7) we obtain, after some algebra, the expression

$$
\begin{aligned}
W & =-\left\langle\bar{\xi}, X_{t_{0}}^{(1)} u\right\rangle \\
= & \int \frac{1}{H_{0}}\left(h \mathbf{v}_{0}+H_{0} \mathbf{v}\right) \overline{\left(h \mathbf{v}_{0}+H_{0} \mathbf{v}\right)} d x d y \\
& +\int\left(\rho_{0}^{\prime}-\frac{\left.\mathbf{v}_{0} \cdot \mathbf{v}_{0}\right)}{H_{0}}\right) \bar{h} d x d y \\
& +\int \frac{\mathbf{v}_{0}}{\partial_{x}\left(R / H_{0}\right)}\left(\omega-\frac{R}{H_{0}} h\right) \overline{\left(\omega-\frac{R}{H_{0}} h\right)} d x d y
\end{aligned}
$$

where $\omega \equiv \hat{\mathbf{z}} \cdot \nabla \times \mathbf{v}$ is the vorticity and $P^{(1)}(h)=P_{0}^{\prime} h$ depends on the choice of model.

The pseudo-energy of a wave is related to (32) by $W|\mathrm{~A}|^{2}$ where $\mathrm{A}$ is the complex amplitude of the wave. If (32) can be both positive and negative we have the possibility of having both positive and negative energy modes which can cause instability in the system (Craik 1985).

The relation (32) is similar to that obtained by Holm et al. (1985) which considered the energy-Casimir method, i.c. a Lyapunov method where the conserved quantities are the energy and the Casimirs. Two dilferences between the methods are worth mentioning. Firstly, in the derivation of their expression Holm et al. (1985) used a Bernoulli function to describe the background flow, but for our sheared background velocity $\mathbf{v}_{0}=v_{0}(x) \hat{\mathbf{y}}$ the equilibrium is 
directly obtained from $\partial_{x} P\left(H_{0}\right)=f(x) \mathrm{v}_{0}(x)$, so no Bernoulli function is needed. Secondly, we make no use of Casimirs, instead we consider dynamical accessible perturbations which allow us to study a less restricted class of equilibria. Casimirs versus dynamical accessible variations have also been discussed by Morrison (1998).

We note that when the background velocity is zero the r.h.s. of (32) is positive definite (assuming $P_{0}^{\prime}>0$ and $H_{0}>0$ ) indicating at least linear stability. When the background velocity is introduced we expect (32) to be indefinite in many cases, thus indicating instability. A sufficient condition for (32) to be positive definite is

$P_{0}^{\prime}-\frac{\mathrm{v}_{0}^{2}}{H_{0}} \geq 0$ and $\frac{\mathrm{v}_{0}}{\partial_{x}\left(R / H_{0}\right)} \geq 0$

In the case of shallow water $\left(P_{0}^{\prime}=g\right)$ the first condition can be written as $\mathrm{v}_{0}^{2} \leq c_{g}^{2}$ where $c_{s}=\left(g H_{0}\right)^{1 / 2}$ is the gravity wave speed. Since the conditions (33) only involve background parameters and no perturbations at all, we know that a flow satisfying (33) will be positive definite for all type of disturbances.

Let us now check how (32) affects the coupling equations (14). First we multiply (14) by $C_{a}, C_{b}$ and $C_{c}$ respectively and then add the complex conjugate to obtain

$$
\begin{aligned}
\frac{d}{d t}\left|C_{j}\right|^{2} & =C_{j} \frac{d}{d t} \bar{C}_{j}+\bar{C}_{j} \frac{d}{d t} C_{j} \\
& =-i \omega_{j} \frac{V}{W_{j}} C_{u} C_{l} C_{t}+i \omega_{j} \frac{\bar{V}}{W_{j}} \overline{C_{a} C_{b} C_{c}}
\end{aligned}
$$

where the overbar denotes complex conjugate.

Next we add the three equations in (34), use the resonance condition (10) and the constancy of $\mathrm{w}_{\mathrm{j}}$. Thus we have

$\frac{d}{d t}\left(W_{a}\left|C_{a}\right|^{2}+W_{b}\left|C_{b}\right|^{2}+W_{c}\left|C_{c}\right|^{2}\right)=0$

If $\mathrm{W}_{\mathrm{a}}, \mathrm{W}_{\mathrm{b}}$ and $\mathrm{W}_{\mathrm{c}}$ all have the same sign then all three terms in (35) are positive and represents three positive energy modes. But if one of $\mathrm{W}_{\mathrm{j}}$ differs in sign we have the possibility of negative energy modes (c.g. Craik 1985) and the squared amplitudes can increase simultaneously for all three waves without violating the conservation of wave energy. But from (32) we see that if we choose a background velocity satisfying (33), then (32) will have the same sign for all types of disturbances, and we thus avoid the explosive behavior that otherwise can be present. When explosive growth occurs, which can bc the case for e.g. Kelvin-Helmholtz systems (Craik 1985), the theory will finally, sufficiently close to the time of explosion, cease to be valid because minor terms neglected in (13) will be important (Weiland and Wilhelmsson 1977). However it is possible in principle to include such terms in the analysis so that a series of quasi-explosive recurrence events occur. In that case the system becomes formally similar to the Fermi-Pasta-Ulam recurrent case. Such an analysis is however outside the scope of this paper since we are essentially talking about sinusoidal waves here and the physics is changed if these are too much deformed.

\section{Summary and conclusion}

We have derived the general coupling coefficients for resonant three-wave interactions in a class of systems containing for example the shallow water model. In the special case of intcraction between two inertial waves and one Rossby wave we have shown that the gencral coupling coefficient could be reduced significally and that the resulting expression contains terms depending on the shear of the background velocity. We have also shown that in some, not too restricted, regimes of approximation those terms are important.

Despite that resonant wave interaction between three nonlinear inertial waves is not possible (they cannot satisfy the resonance conditions (10) and (17)) it is still possible to transfer energy between inertial waves by using the coupling described above. Here we thus use the Rossby wave to transfer energy between the inertial waves.

From another point of view this coupling is a nonlinear process to move energy from two interacting waves, with frequency close to the frequency of planetary rotation, down to a Rossby wave with a much lower frequency. Since the Rossby wave may couple with two other Rossby waves we thus have a mechanism to remove energy from frequencies close to the Coriolis frequency. This might be important in atmospheric and ocean research where many energy spectra seem to have a peak close to that frequency. We have also looked at the stability of the system by using a conserved pseudo-energy expression obtained from the Hamiltonian theory. The result here is a condition on the background velocity which allows us to avoid the explosive behavior that can occur.

Acknowledgements: The author is grateful for discussions with Prof. Lennart Stenflo and Dr. Jonas Larsson and for the suggestions they, as well as an anonymous referee, have made.

\section{References}

Axelsson, P., Threc-wave coupling in a stratified MHD plasma, Nonlin. Proc. in Geophysics, 5, 105-110, 1998

Craik, A.D.D., Wave interactions and fluid flows, Cambridge University Press, 1985

Dowling, T.E., Dynamics of Jovian atmospheres, Annu. Rev. Fluid Mech., $27,293-234,1995$

Fubrikant, A.L. and Stcpanyants, Yu.A., Propagation of waves in shear flows, World Scientific Publ., 1998

Holm, D.D, Marsden, J.E., Ratiu, T. and Weinstein,A., Nonlinear stability of fluid and plasma equilibria, Phys. Rep., 123, 1-116, 1985

Horton, W. and Ichikawa, Y-H., Chaos and structures in nonlinear plasmas, World Scientific Publ., 1996

Larsson, $\mathrm{J}$., A new Hamiltonian formulation for fluids and plasmas, $I$. Plasma Phys., 55, 235-259, 1996

Larsson, J., Hamilionian Theory for waves and the resonant three-wave interaction process, Phys. Scripta, T75, 173-175, 1998 a

Larsson, J., The Hamiltonian-adjoint equation and perturbation theory, Phys. Scripta, 58, 97-101, 1998b

Morrison,P. J., Hamiltonian description of the ideal tluid, Reviews of modern physics, 70, 467-521, 1998 
Nezlin, M.V. and Chernikov, G.P.,Analogy between drift vortices in plasma and geophysical hydrodynamics, Plasma Phys. Report, 21, 922-944, 1995

Petviashvili,V. and Pokhotelov, O., Solitary waves in plasmas and in the atmosphere, Gordon and Breach, 1992

Pokhotelov, O.A., McKenzie,J.F., Shukla, P.K. and Stenflo, L., Nonlincary coupled inertial and Rossby waves, Phys. Fluids, 7, 1785-1787, 1995

Pokhotelov, O.A., Stenflo, L. and Shukla, P.K., Nonlinear interaction of electrostatic ion-cyclotron and drift waves in plasmas, J. Plasma Physics, 55, 187-191, 1996

Salmon, R., Semigeostrophic theory as a Dirac-bracket projection, J. Fluid Mech., 196, 345-358, 1988

Van Delden, A., The dynamics of meso-scale atmospheric circulations, Phys. Rep., 211, 251-376, 1992

Weiland, J. and Wilhelmsson, H., Coherent non-linear interaction of waves in plasmas, Pergamon Press, 1977

Zakharov, V. E., Hamiltonian formalism for hydrodynamic plasma models, Sov. Phys.-JETP, 33, 927-932, 1971

Zakharov, V.E., Kolmogrov spectra in weak turbulence problems, Handbook of plasma physics, 2, 3-36, 1984 